\title{
Virtual Reality as an Environment for CBR
}

\author{
Ian Watson \& Leonardo Oliveira \\ AI-CBR, University of Salford, Bridgewater Building, Salford, M5 4WT \\ Tel: +44 161-295-5227 \\ Fax: +44 161-295-5011 \\ www.ai-cbr.org \\ ian@ai-cbr.org
}

\begin{abstract}
This paper presents the results of an investigation into the suitability of a Virtual Reality (VR) environment for case-based reasoning (CBR). The paper will show that for problem domains where visualisation of the case is important (e.g. in design or training applications) VR environments have great potential. A prototype has been developed as part of this research. It holds past experiences of experts in the inspection of health and safety regulations of scaffold structures. Each case in the casebase describes a virtual scaffold structure along with the various tasks involved in its inspection. In order to encourage further applications of this approach, this paper describes features of VR that potentially make VR a powerful tool for CBR.
\end{abstract}

\section{Introduction}

In many applications of CBR the visualisation of the cases is critically important to the success of the system. This is apparent with case-based design applications since designers typically interact visually with their work. This is also true for many training and education systems. To date almost all case-based design and case-based training systems that need to show the user an image, CAD plan or movie have used a linked multimedia approach [1 \& 2].

In these systems cases are created that abstract features from the original media. For example, in a case-based architectural design system buildings may be described by a case representation of features describing the size, shape, purpose, construction, orientation, etc. of buildings. Each individual case may then have links to multimedia files such as CAD plans of the building, and photos or videos.

This linked multimedia approach has severe limitations for CBR. In particular adaptation of a retrieved design becomes problematic, because whilst adaptation may be possible at the case feature level adaptation of the linked multimedia files is either impossible (in the case of photos or videos) or extremely difficult (in the case of CAD plans). Consequently, although the design may have been conceptually adapted (i.e., adapted at the abstract feature level) the users are unable to see a visualisation of the adapted solution. Attempts have been made to overcome this. Typically this involves creating a system that retrieves and adapts schematics of designs - since these are much simpler and easier to manipulate than full designs. Or, users interact with the system through a CAD interface that visualises cases as in 
the CADRE project [3]. A novel approach was taken in one aspect of the FABEL project which directly compared abstracted bitmaps of design elements [4]. A full review of case-based design systems can be found in [2].

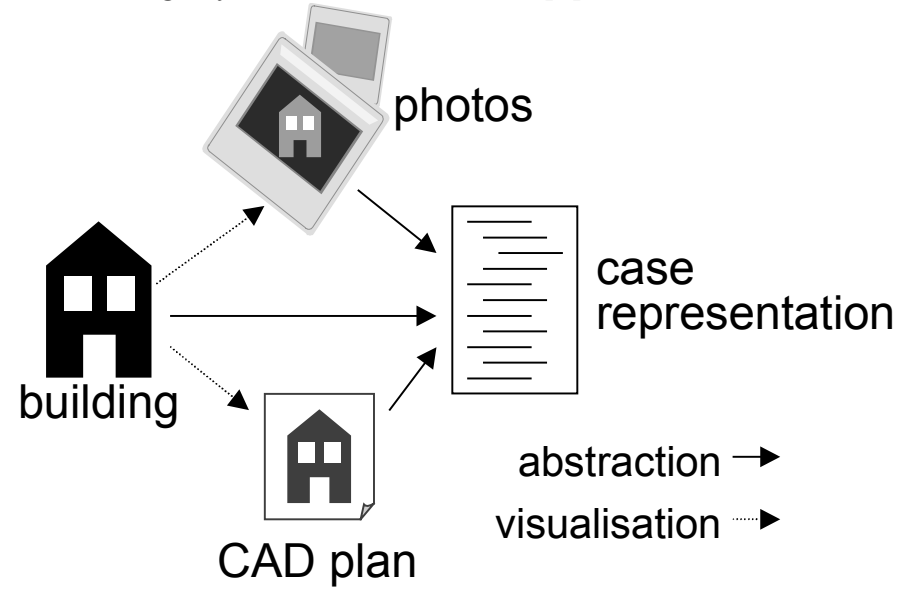

Figure 1. Linked Multimedia Case Representation

Increasingly designers are using VR to visualise designs since virtual environments provide many useful features to designers such as a $3 \mathrm{D}$ representation, walk-throughs and clash detection [5]. On investigation it becomes apparent that VR offers unique advantages to CBR systems. Within a virtual environment it is possible to create a unified representation with no need for multimedia file attachments or a mediating CAD interface. An object in a virtual environment has features such as: position, size, shape, colour or rendering, and motion. In addition user defined features may be added. A CBR system can use these features to assess similarity, retrieve and if required perform adaptation. The virtual environment automatically provides the visualisation.

For example, consider a simple blocks world example. In VR it is possible to populate a virtual blocks world with $3 \mathrm{D}$ shapes such as cubes, spheres, pyramids and cylinders of different colours, sizes and orientations, as in Figure 2. A user could select any object and ask the system to find a similar object anywhere in the virtual world.

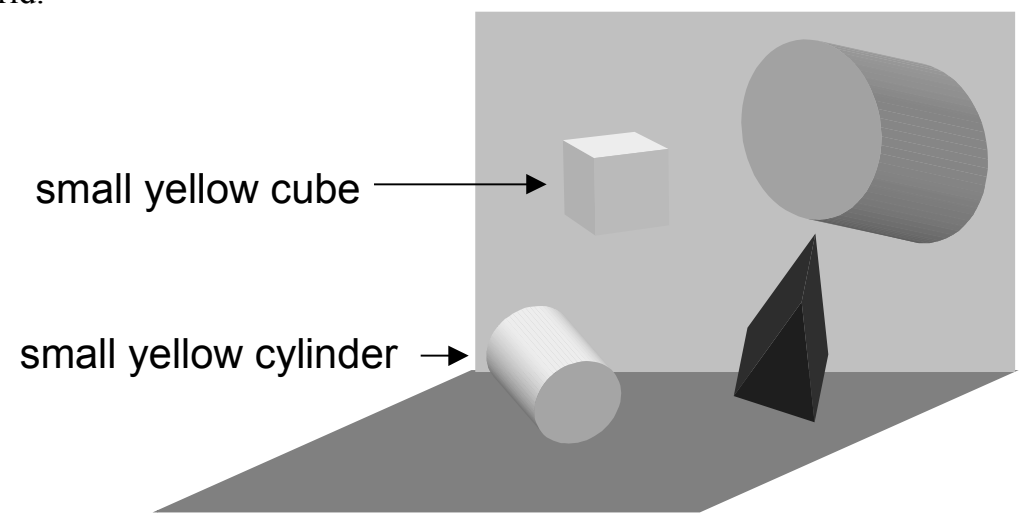

Figure 2 Objects in a Virtual Blocks World 
Let us assume the user has selected a small yellow cylinder. If there are no other small yellow cylinders in the virtual blocks world the system might retrieve a small yellow cube as the closest match because it is a similar colour, has a similar volume and is in a similar spatial orientation. The large cylinder (to the top right in Figure 2) has been rejected because its colour doesn't match, its volume is different and its orientation is different. (note that assessing similarity in a $3 \mathrm{D}$ virtual world will not necessarily be straightforward). The system could then copy the retrieved object and adapt it by changing its shape to a cylinder. This new object would be visualised in the world as soon as it was created. A demonstration of this principle can be viewed at: http://www.surveying.salford.ac.uk/postgrad/leo/resf.htm (this requires the Viscape VR plug-in which can be downloaded from: http://www.superscape.com/download/viscape/).

Thus, VR enables us to create a unified case representation and visualisation environment within which we can describe objects, assess similarity, retrieve and adapt and most crucially instantly visualise the results of any adaptation.

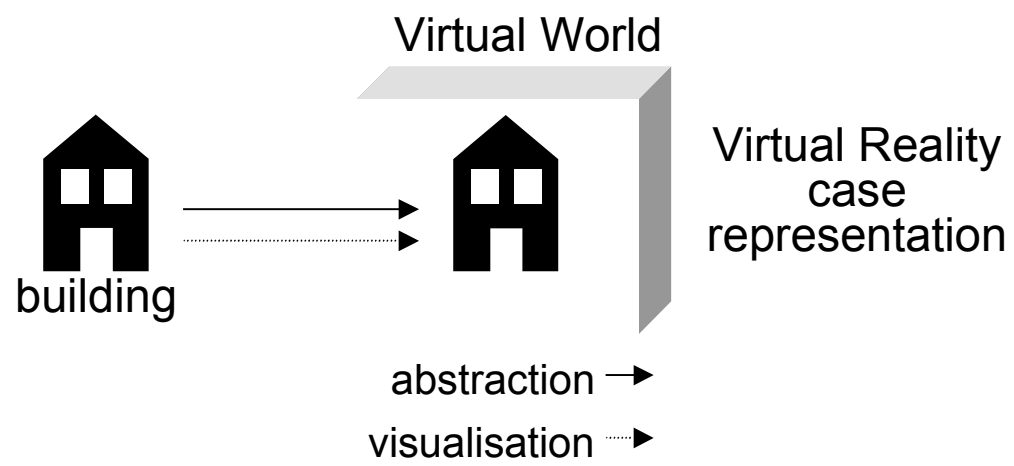

Figure 3. Unified Case Representation

Our research suggests that VR could substantially improve many CBR systems. VR is a technology that handles representations of the physical world [5] enabling the communication of complex ideas [6]. Its capacity to handle objects and their properties, to walk-through the virtual environment in real time, and to simulate the real world in a 3D graphical display, makes this technology an ideal computer tool for describing real world models [7]. Bringing together VR and CBR, within a unified framework was the main objective of this research.

\section{The CBR Interface}

Dearden [8] stated that:

"the success of any interactive intelligent system, whether it is rule-based or case-based, is dependent not only on the quality or on the appropriateness of the knowledge encapsulated within the system but also on the quality of the interaction that the system supports".

Apart from some academic demonstrators, the interface of a CBR system plays an important role in the quality of the support provided to users. Users have to at least 
interface with a CBR system to input a problem description and to receive the information contained in any retrieved cases.

Most current CBR systems use feature based description of problems [9]. Several CBR systems can be found using multimedia techniques, such as sounds, pictures, image animation and even digitised films [10]. However, cases represented by these types of visualisation media have characteristics which are approached quite differently in our research. Firstly, such images are not understood or evaluated by the CBR system, since there is no language to access the internal contents of the files. Moreover, any case adaptation would require the adaptation or recording of new images and would not be based on the features of the previous image. An example of a learning application of CBR using digitised videos for case representation is the SPIEL (Story Producer for Interactive Learning) system [11]. Case features in SPIEL are textual descriptions of videos and these are used to retrieve the video material.

Such visualisation techniques present static information. Note that the term static refers to the information content only, and has no relation to the way that information is displayed to users. A movie is static because the content of each frame always remains the same. This definition also applies to animated images and photos. Conversely, VR is an active medium in the sense that users can interact with it by performing actions such as moving through the virtual world or moving objects. VR is also active in the sense that it can be programmatically changed; for example changing the colour of an object from red to blue. Thus, VR can increase CBR's potential to provide design and training applications as close to real situations as possible.

\section{The "Art of Memory"}

"Images must be lively, active, striking, charged with emotional affects so that they may pass through the door of the storehouse of memory... however, we need to ask ourselves what would constitute the lively, active, striking and emotionally charged equivalents for our own time." [12, p.286]

This section draws upon Yates' book "Art of Memory", focusing on the main points of interest for this research work. An assumption of this work is that people remember things in the context of place. An example provided by Yates describes someone who was called upon to name unrecognisable victims of a vehicle accident. This person was able to name the victims only by recalling the places where they had been seated. Space is a powerful trigger to recall memories and the information associated to them. Some other major issues that the Yates offers to support the use of VR for memory recall are listed below.

- imaginary or real structures - a building (e.g. the Eiffel Tower) is an example where a textual or feature based description would never provide the same level of remembrance as the visual representation of the structure.

- concreteness and memorability - recalling a view of a space is easier than recalling abstract symbols (such as abstract concepts or pieces of language). Concreteness produces memorability which is a key factor for learning.

- taxonomies for thought - spaces have a coherence and logic which can be used to connect one idea to another, becoming a prominent tool to help user's mnemonic thought. 
- representing reality - although some aspects of reality only exist inside the head of the individual, visual designs can be used for mediation between individuals.

- motion detection - is a strong element in visual perception and users can gain much information from it. Moreover, motion is the main source of understanding for many domains such as driving.

To conclude, virtual worlds have much to offer by helping designers or learners understand and recall information.

\section{Designing Cases for VR}

In addition to the usual issues of case representation in CBR, such as feature selection and indexing cases for retrieval, this research also inevitably involved the construction of the virtual worlds within which the cases are represented. This is a development process with no universally accepted methodology to follow.

Deciding what can be represented in VR is not the only issue to consider. For instance, some factors such as the user's interaction with the virtual world, and the way it will be displayed should also be carefully evaluated [13]. In order to help those interested in representing cases in VR, a comparison of the representational ability of three VR software systems was undertaken, the results of which can be seen at the project's web site:

(http://www.surveying.salford.ac.uk/postgrad/Leo/vraspects.html).

Thus, developers can match their needs to the VR package whose capabilities best suits their application. The VR packages compared were:

- $\quad$ Superscape VRT version 5 (http://www.superscape.com)

- $\quad$ Sense 8 WTK version 6; (http://www.sense8.com); and

- Integrated Data Systems Inc. IDS V*Realm Builder (http://www.ids-net.com)

We only considered built-in functions of the VR packages, avoiding the need for programmers to include those features in the applications. Most VR systems provide a programming language and thus many additional features can be programmed in. However, if the aspect requires programming, it has not been included as supported in our comparison. Developers should consider the following before choosing a VR tool for case representation:

Even when a case representation involves some spatial attributes, developers should ask themselves whether a 3D graphics display would enhance understanding.

The creation of virtual worlds is a time-consuming task, even though libraries of components can be built up to accelerate the process. Developers should be aware of this factor. Most of the work involved in building virtual worlds is uninteresting, repetitive and requires long hours of debugging and optimising.

There is a danger that the same virtual world when running on different hardware may appear differently from the developers original intention.

In addition, questions have been raised regarding the loss of abstraction that VR entails and its possible counter productive effect on understanding in certain domains [13 \& 14]. For instance, Satalich [14] describes a study where the users of VR performed worse than a group who only worked on paper. The same author cited that there are several reasons supporting these results, such as: the amount of time users have been using VR, considering the novelty of the technology; the issues and the subject evaluated; and the deficiencies of the hardware used. We suggest it 
is worth keeping these results in mind. However there is no reason to consider computer systems involving VR as necessarily inferior to traditional learning systems [14].

\section{Developing the Prototype}

A prototype has been developed as part of this research to explore the issues involving VR as an environment for CBR. The following sub-sections provide further details on the development of this prototype.

\subsection{Development Software}

The VR software used for the development of this application was Superscape VRT version 4.0. It incorporates an environment for building VR worlds and a programming language that allowed the development of our CBR system. The creation of virtual worlds is not an easy task. One of the main lessons learnt from the implementation stage was the importance of using modelling techniques prior to the representation of the cases in the VR environment. Modelling techniques were also used for such tasks as: understanding the statements provided by the experts, helping to establish the sequence of the training sessions and representing the cases in VR.

Modelling the information in each case and the relationships between the information prior to implementation proved to be helpful in saving time building the virtual environments. The modelling technique used in this work was Express $G$ and Figure 4 shows one of the models used to implement a case in VR (i.e. building a scaffold structure in accordance to British Standard BS 5973-1990).

\subsection{Case Memory Structure}

The structure for the case memory in the prototype was implemented using the concept of Memory Organisation Packets (MOPs) and Scripts, described in [15 \& 16]. This concept says, for instance, that a construction site with a scaffold structure serves as a MOP for an expert, and that the activities involved in its inspection constitute Scripts. Thus, each examination of a scaffold is represented as a series of Scripts (or tasks) which may be common for other types of scaffold structures.

For example, one of the cases present in the prototype describes a site in which repairs will be done on the roof of a three-storey building. In order to identify whether the scaffold complies with British Standard BS 5973-1990, certain tasks have to be performed. One of these tasks is to check whether the vertical bars (technically called standards) are well centred on top of soil plates. Any scaffold structure (which is not suspended), must have its bases well centred on top of soil plates. Thus, the task of inspecting if standards are properly supported by the soil plates is an example of a Script common to several MOPs.

Each case (or MOP) in the prototype has been represented in a different VR file which also contains the Scripts belonging to each case. This approach was adopted because of the very large number of objects involved in the representation of scaffold structures but it does slow down the interaction process. The internal structure of the case files follows an object-oriented hierarchy. This hierarchical structure serves as an organisational foundation upon which to build virtual worlds. It supports the inheritance of features down the hierarchy. Inheritance is useful for several reasons. For instance, when an object is child of another object, its position within the virtual world will them be defined in terms of the position relative to the 
parent, rather than in terms of its world co-ordinates position. This characteristic of inheriting properties is specially important in this work, because it supports case adaptation, where full hierarchies of objects can be used to create new cases by feature substitution.

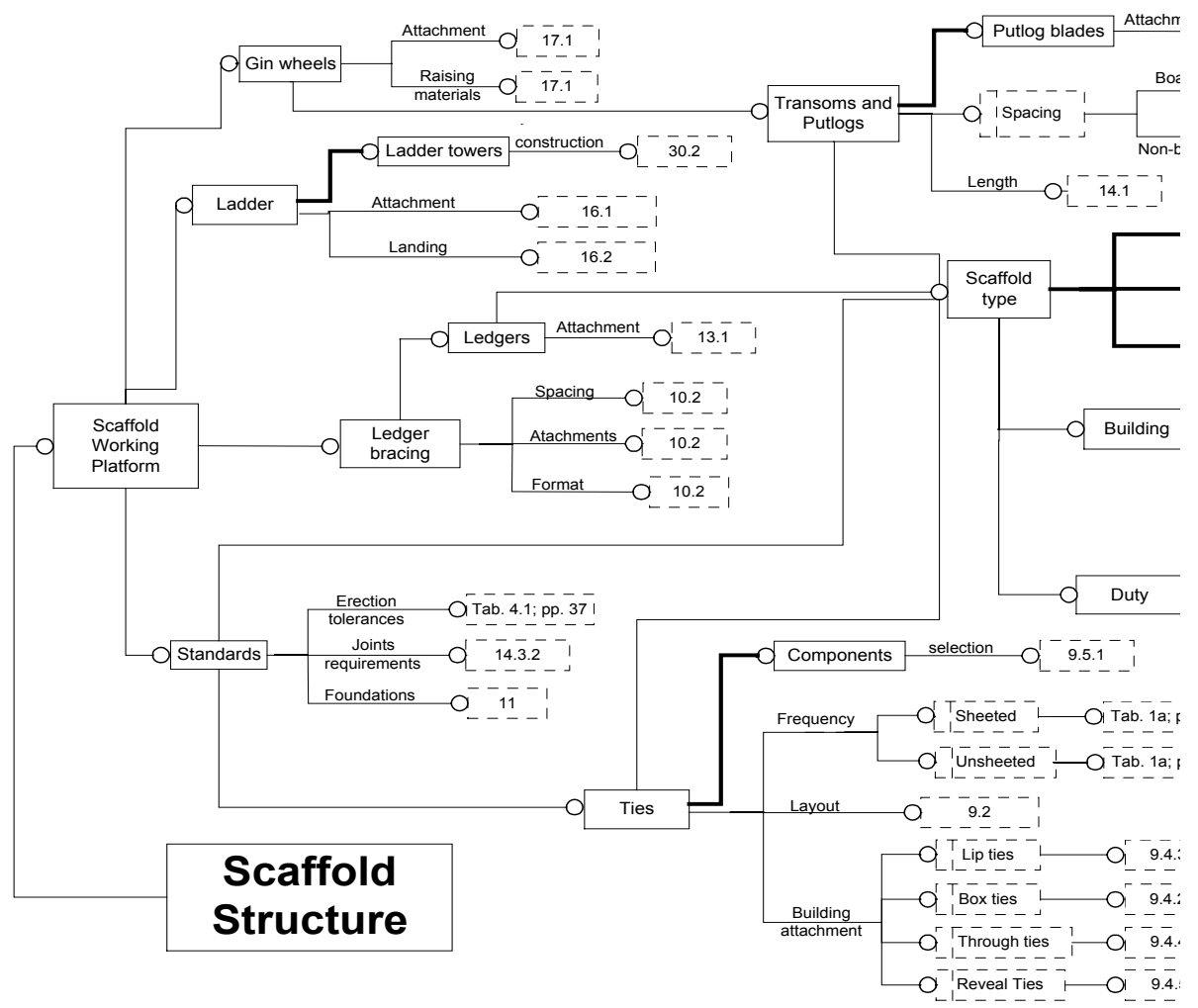

Figure 4. A section of the Express G Case Model

\subsection{Identifying Case Features}

Feature identification in the prototype results from the combination of two main issues: indexing features that differentiate the cases in the repository and properly identify them for retrieval; and the capabilities of the object-oriented hierarchy used to represent the cases in VR. Redmond [17] stated that CBR applications for training should include two aspects; namely, presenting the same kind of situations users encounter at work, as well as giving a presentation that will be retained in a user's memory. The same author indicates that one of the greatest challenges in building such systems is the ability to provide features capable of proper case retrieval as well as helping users to access case knowledge in real situations.

In the light of this feature identification was approached in terms of describing scaffold structures and the tasks involved in performing inspections for health \& safety regulations. Thus, features were provided at two levels: at the top level describing the cases, and at a lower level describing the Scripts each case contains. Case features have been chosen in terms of features that differentiate the cases contained in the repository, such as the type of scaffold, the type of work to be 
provided, type of building, scaffold dimensions and site characteristics. Script features concern the description of the items and tasks to perform inspecting the scaffold structure. Both descriptions were carried out in close contact with experts, who also provided guidelines for the task sequence to be followed performing scaffold inspection.

As discussed before, VR provides direct access to the contents of the visualisations. Some advantages that can be taken from accessing the contents of the files are:

- new cases can be automatically created or existing ones adapted by combining objects from other virtual cases; and

- libraries containing hierarchies and objects can be shared between users and developers to speed up the process of case representation.

Currently the prototype does not perform automatic adaptation or creation. The main reasons for these limitations are the restricted time for the development of this research as well as the complexity that training applications require, specially forbidding inexperienced users to insert inappropriate cases in the repository.

\subsection{Retrieval Method}

The method for retrieval used in the prototype allows users to search only for MOPs, only for Scripts or for both together. This is possible because cases and Scripts are independent (see section 5.3). The retrieval mechanism performs a search based on a weighted nearest-neighbour approach [18], and the core of the retrieval algorithm is shown in Figure 5. The code of the algorithm has been written in SCL (Superscape Control Language.

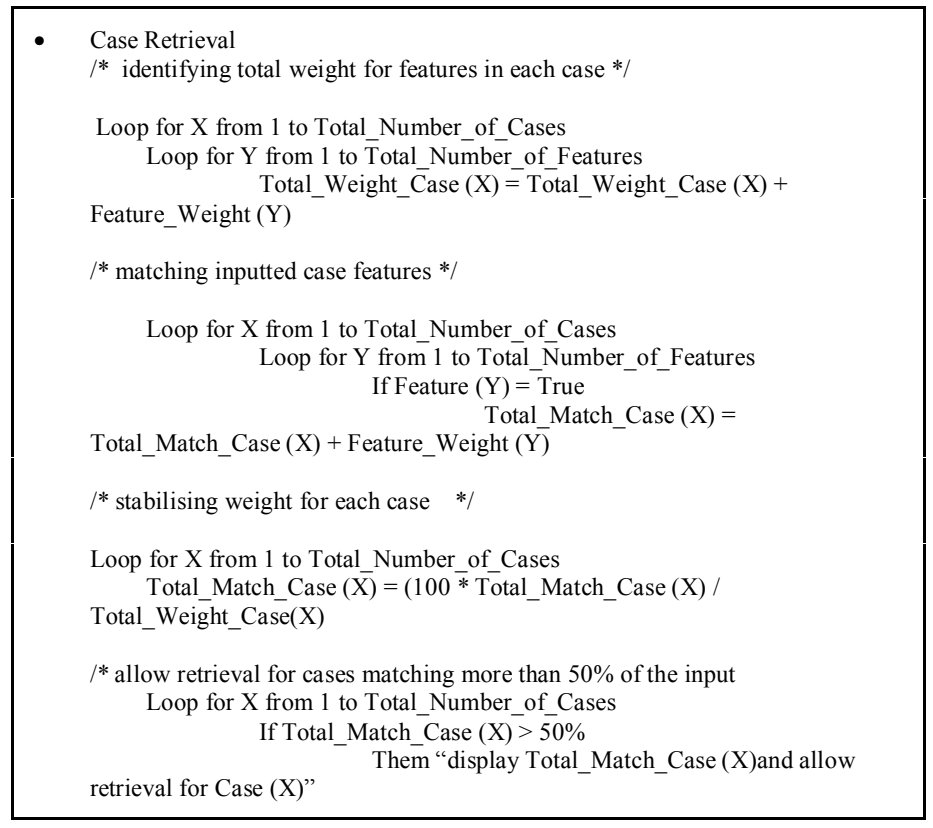

Figure 5. Part of the Retrieval Algorithm 


\subsection{Training Performance}

On real construction sites, experts do not follow a pre-established sequence to inspect health \& safety regulations on scaffold structures (although some prescriptions do exist). The normal approach is based on checking key parts of the structure, which allows experts to identify whether the structure has been properly erected, will be safe to work on, and safe for anyone in the vicinity of the structure. Obviously the inspector does not want to climb a dangerous scaffold structure and it is difficult and dangerous to show trainees unsafe scaffolds.

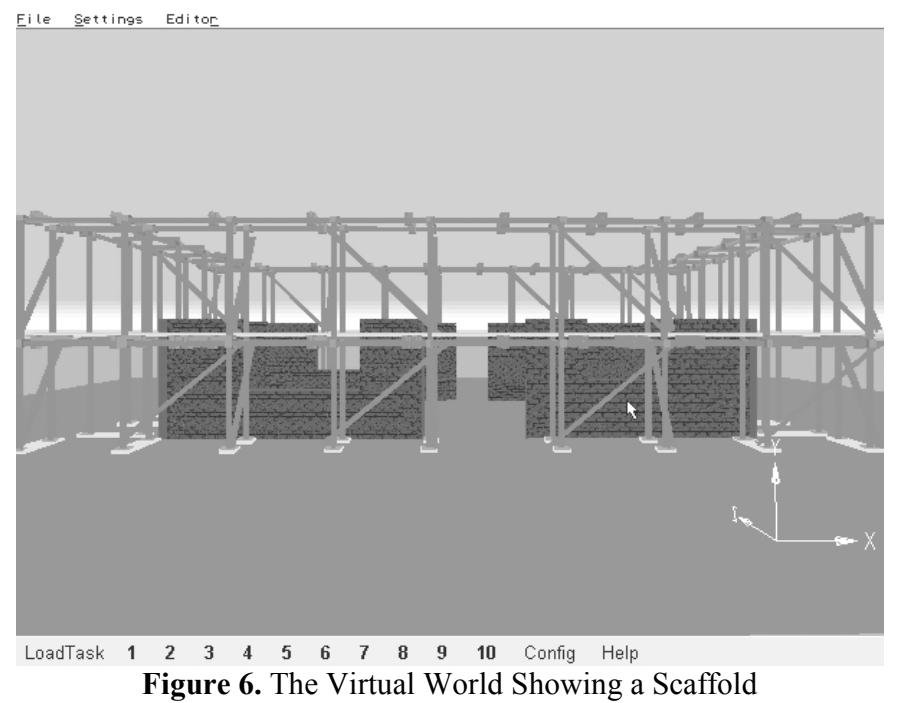

The system allows users the freedom to retrieve the case they want, according to the nature of their problem. The prototype has also been designed to cater for different levels of users, such as: beginners who may blindly follow the system's guidelines for the case retrieval sequence; experienced users who retrieve the script they want to reinforce their knowledge; and trainers who can illustrate their lessons with a virtual representation of past occurrences on site.

Figure 6 shows one of the cases present in the case library. The menu bar at the bottom prescribes a sequence of scripts associated with the inspection of health \& safety regulations on this structure. When clicking the mouse over the script number, the system presents guidelines on how to properly perform the task associated with the script. The number will change its colour on the menu bar, indicating that the task has been performed.

For example, one of the scripts performs inspection of the overhang at the end of scaffolding boards. The system moves the viewpoint around the structure, replicating the views that an expert would have on a real site (see Figure 7). Theoretical information about this task is also provided by reading the menus or listening to an expert's recorded advice. Most of the objects of the scaffold structure hold some additional information regarding dimensions, material nomenclature, etc. This information can be accessed by clicking on the right mouse button on top of the virtual object. Thus, each case works as a repository of information concerning the domain of inspection of health \& safety regulations on scaffold structures. 


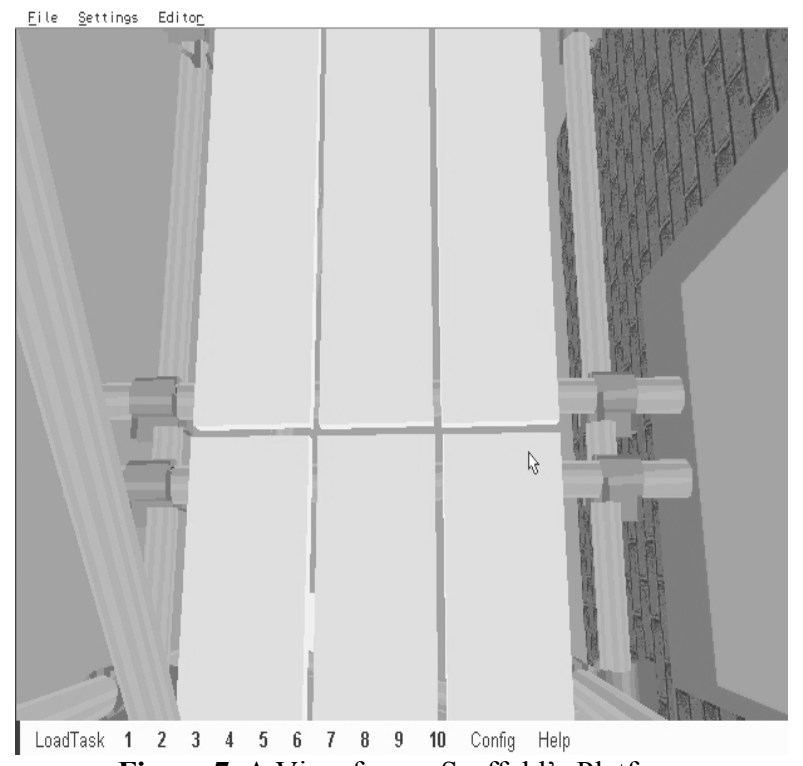

Figure 7. A View from a Scaffold's Platform

Users can also freely walk-through the virtual case searching for irregularities on the structure independently from the system's guidelines. Once an irregularity is found, users can click on the object and the system will fix the irregularity. This approach is specially important for trainers, who can use the system as a tool to illustrate site occurrences. For instance, viewpoints, such as that presented in Figure 8, could be difficult or dangerous to access on real structures.

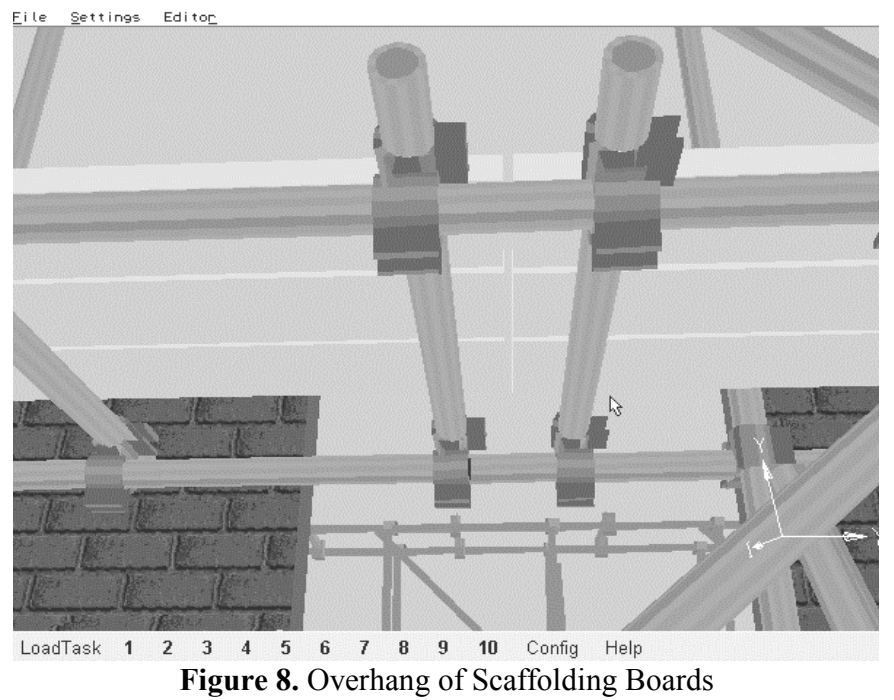

Moreover, trainees would need to physically go to a site where this structure is present, and special supervision would be required. 


\section{Conclusions}

It is no longer necessary to create disjointed CBR systems that abstract features from the visual world into separate case representations linked to multimedia files. VR provides a powerful environment within which case representation, retrieval, and adaptation can be unified with visualisation. VR could well be the breakthrough that those creating case-based design systems have been looking for. For educational systems VR also has potential. Experiencing is central to using VR as a visualisation tool and is a dominant characteristic of our prototype. VR can support learning by doing, since the user can perform actions within the learning environment. Thus, the very tangible world simulations achieved by VR can provide an interactive environment that helps understanding of the lessons displayed.

The process of designing virtual worlds can be difficult and timeconsuming. However, software for virtual world building is becoming more powerful and easier to use. Moreover, third-party objects are increasingly becoming available which will ease the task of virtual world design. The use of modelling techniques to make explicit objects' behaviour, dimensions, positions, dependencies, and links with other objects will help speed up world creation. Thus, if your CBR application would benefit from a visualisation of the cases you should consider using a VR environment as your implementation environment since VR can provide a unified case representation and visualisation environment. Future work will involve developing a VR case-based design system, combining VR and CBR for planning, and a fundamental investigation of the notion of similarity within virtual worlds.

\section{References}

[1] Maher, M.L., Balachandran, B., \& Zhang, D.M. (1995). Case-Based Reasoning in Design. Lawrence Erlbaum Associates, Publishers. USA.

[2] Watson, I. \& Perera, R.S. (1997). Case-based design: A review \& analysis of building design applications. Artificial Intelligence for Engineering Design, Analysis \& manufacture, 11: pp.59-87

[3] Smith, I.F.C. \& Faltings, B.V. (1994). Spatial Design of Complex Artefacts using Cases. In, Proc. 10th. Conf. AI for Applications, pp.70-76.

[4] Coulon, C.H., \& Steffens, R. (1994). Comparing fragments by their images. In, Similarity Concepts and Retrieval Methods. Vob, A. (Ed.). pp.36-44. GMD Sankt Augustin.

[5] Earnshaw, R.A.; Gigante, M.A. and Jones, H., (1993). Virtual Reality Systems. Academic Press.

[6] Sherman, W.R. and Craig, A.B. (1995). Literacy in Virtual Reality: a New Medium. Computer Graphics, Vol. 29 (4), pp. 37-41.

[7] Brooks, M.R. (1994). Virtual reality interfaces for complex environments. M.Sc. dissertation, University of Manchester.

[8] Dearden, A.M. (1995). Improving the interfaces to interactive case memories. In, Progress in Case-Based Reasoning. Ed. Watson, I. LNAI \#1020, pp.73-84. Springer-Verlag, Berlin.

[9] Watson, I., (1997). Applying case-based reasoning: techniques for enterprise systems. Morgan Kaufmann Publishers. San Francisco, CA.

[10] Maher, M.L., \& Balachandran, B. (1994).Multimedia approach to case-based structural design. The journal of computing in civil engineering, 8(3): pp.359-376. 
Watson, I. \& Oliveira, L. (1998). Virtual Reality as an Environment for Case-Based Reasoning.

In, Advances in Case-Based Reasoning. Cunningham, P. \& Smyth, B. (Eds.), pp.448-459.

Lecture Notes in Artificial Intelligence \# 1488. Springer-Verlag, Berlin. ISBN 3-540-64990-5

[11] Burke, R. and Kass, A. (1996) - Retrieving stories for case-based teaching. In: Leake, D.B. (ed.) - Case-based reasoning: experiences, lessons and future directions. AAAI Press/MIT Press, pp. 93-109.

[12] Yates, F.A, (1966). The art of memory. Routledge, London

[13] Bolter, J.D. (1986). The Turing Man: western culture in the computer age. Penguin Publishers.

[14] Satalich, G.A. (1995). Navigating and wayfinding in virtual reality: finding proper tools and cues to enhance navigation awareness. Masters Thesis, Human Interface Technology Lab, University of Washington, http://www.hitl.washington.edu/publications/satalich/ (last visited 05/97).

[15] Schank, R.C., (1982). Dynamic memory: a theory of reminding and learning in computer and people. Cambridge University Press.

[16] Schank, R.D., \& Riesbeck, C.K., 1994. Inside case-based reasoning. Lawrence Erlbaum Associated, Inc. Publishers. Hillsdale, New Jersey.

[17] Redmond M.A. 1992 - Learning by observing and understanding expert problem solving. Georgia Institute of Technology, College of Computing. Technical Report No . GIT-CC-92/43, Atlanta.

[18] Kolodner, J.L., (1993). Case-based reasoning. Morgan Kaufmann, San Francisco, California.

further information on all aspects of case-based reasoning can be found at www.ai-cbr.org 